\title{
The Role of Glucagon-Like Peptide 1 Receptor Agonists and Sodium-Glucose Cotransporter 2 Inhibitors in Reducing Cardiovascular Events in Patients with Type 2 Diabetes
}

\author{
Gwang Sil Kim ${ }^{1,2}$, Joong Hyun Park ${ }^{3}$, Jong Chul Won ${ }^{1,2}$ \\ ${ }^{1}$ Department of Internal Medicine, Inje University Sanggye Paik Hospital, Inje University College of Medicine, Seoul; \\ ${ }^{2}$ Cardiovascular and Metabolic Disease Center (CMDC), Inje University, Busan; ${ }^{3}$ Department of Neurology, Inje University \\ Sanggye Paik Hospital, Inje University College of Medicine, Seoul, Korea
}

The prevalence of type 2 diabetes mellitus (T2DM), which is associated with cardiovascular morbidity and mortality, is increasing worldwide. Although there have been advances in diabetes treatments that reduce microvascular complications (nephropathy, neuropathy, retinopathy), many clinical studies have found that conventional oral hypoglycemic agents and glucose control alone failed to reduce cardiovascular disease. Thus, incretin-based therapies including glucagon-like peptide 1 (GLP-1) receptor agonists (RAs) and sodium-glucose cotransporter 2 inhibitors (SGLT-2Is) represent a new area of research, and may serve as novel therapeutics for treating hyperglycemia and modifying other cardiovascular risk factors. Recently, it has been confirmed that several drugs in these classes, including canagliflozin, empagliflozin, semaglutide, and liraglutide, are safe and possess cardioprotective effects. We review the most recent cardiovascular outcome trials on GLP-1RAs and SGLT-2Is, and discuss their implications for treating patients with T2DM in terms of protective effects against cardiovascular disease.

Keywords: Diabetes mellitus; Heart failure; Hypoglycemic agents; Myocardial ischemia

\section{INTRODUCTION}

The prevalence of type 2 diabetes mellitus (T2DM) is increasing globally [1]. Although the prognosis of patients with T2DM has improved, the associated cardiovascular mortality and morbidity pose a considerable challenge for healthcare systems [2]. The risk of cardiovascular disease (CVD) is two to four times higher in patients with diabetes than in their non-diabetic counterparts [3]. In addition to glucose control, preventing CVD in these patients is essential [4].

Although intensive glucose control has been shown to reduce microvascular complications [5], controversy remains as to whether it reduces macrovascular complications [6,7]. The negative effects of glucose-lowering agents in patients with an increased risk of heart failure (HF) became evident after rosiglitazone, a thiazolidinedione, was withdrawn from the European Union market due to evidence of increased risk of CVD, including myocardial infarction (MI) [8]. In response, the U.S. Food and Drug Administration and the European Medicines Agency began requiring hypoglycemic therapies to demonstrate an acceptable cardiovascular risk profile [9].

Recently, several drug classes have demonstrated a significant
Received: 15 January 2019, Revised: 25 February 2019,

Accepted: 4 March 2019

Corresponding author: Jong Chul Won

Department of Internal Medicine, Inje University Sanggye Paik Hospital, Inje University College of Medicine, 1342 Dongil-ro, Nowon-gu, Seoul 01757, Korea Tel: +82-2-950-8860, Fax: +82-2-2091-1464, E-mail: drwonjc@gmail.com
Copyright $(\underset{2019}{2}$ Korean Endocrine Society

This is an Open Access article distributed under the terms of the Creative Commons Attribution Non-Commercial License (http://creativecommons.org/ licenses/by-nc/4.0/) which permits unrestricted non-commercial use, distribution, and reproduction in any medium, provided the original work is properly cited. 
reduction in major adverse cardiovascular events (MACE), death, and hospitalizations for HF (HHF) [10-14]. These include incretin-based therapies, such as glucagon-like peptide 1 (GLP-1) receptor agonists (RAs) and sodium-glucose cotransporter 2 inhibitors (SGLT-2Is). Based on these findings, the recently published guidelines of the American Diabetes Association (ADA) and the European Association for the Study of Diabetes (EASD) recommend either SGLT-2Is or GLP-1RAs in patients with T2DM who cannot achieve their target level of glycemic control with metformin [15].

We review the most recent cardiovascular outcome trials (CVOTs) of GLP-1 receptor agonists (RAs) and SGLT-2Is, and discuss their implications for treating patients with T2DM in terms of cardioprotective effects.

\section{CARDIOVASCULAR EVENTS IN PATIENTS WITH T2DM}

\section{Atherosclerosis: epidemiology and pathogenesis}

Atherosclerosis is one of the most frequently fatal complications in patients with T2DM [2]. The prevalence of coronary artery disease $(10.3 \%)$ and stroke $(6.7 \%)$ in Korea is more than twice as high in patients with T2DM than in the general population [16], and mortality in patients with CVD is more than three times higher [17]. In patients with T2DM, chronic hyperglycemia, elevated levels of low density lipoprotein cholesterol and triglycerides, and an increased inflammatory response are associated with atherosclerosis [18]. In addition, patients with diabetes may have other CVD risk factors, such as hypertension, dyslipidemia, obesity, physical inactivity, chronic kidney disease (CKD), and smoking. Previous studies have suggested that concomitant control of other CVD risk factors is important for glucose control, as well as for reducing CVD events and death $[19,20]$. Although strict glycemic control is associated with a reduced incidence of microvascular complications, the impact of glucose control on macrovascular complications is less well understood [21]. Newer drugs have advantages with respect to addressing CVD risk factors, and thus could decrease the rate of CVD events.

\section{Heart failure: epidemiology and pathogenesis}

Derangement of cardiac glucose metabolism in patients with diabetes is associated with structural and functional abnormalities of the heart, which result in HF; thus, the risk of HF is increased two- to five-fold in patients with diabetes compared to those without diabetes [22]. Among Korean patients with HF, 49.1\% had diabetes [23]. However, there is a general lack of data regarding the prevalence of HF in patients with diabetes in Korea.

The precise mechanism by which hyperglycemia impairs cardiac contraction is still unknown. However, increases in free fatty acid oxidation, oxidative stress, and mitochondrial dysfunction, as well as impaired glucose utilization in cardiac myocytes, seem to be associated with poor systolic and diastolic contractile capacity, even in patients without atherosclerotic coronary artery disease $[24,25]$. In addition, impaired microvascular endothelial function, increased myocardial fibrosis, activation of the renin-angiotensin system, and sympathetic overactivity also contribute to HF [24]. Although it has been suggested that hyperglycemia is a critical trigger of HF, not all hypoglycemic agents have a protective effect against HF, due to hyperinsulinemia, water retention, and decreased utilization of glucose by cardiac myocytes. Certain hypoglycemic agents are associated with an increased risk of HF, such as rosiglitazone, a thiazolidinedione [26]. Excessive glucose lowering was correlated with HF in the United Kingdom Prospective Diabetes Study [27], and a meta-analysis of 13 studies $(n=34,533)$ revealed that that intensive glucose control resulted in a $47 \%$ increased risk of HF $(P<0.001)$ [28]. Therefore, for effective management of hyperglycemia in patients with an increased risk of HF, clinical data informing the choice of hypoglycemic agents and target blood glucose levels are required.

\section{RECENTLY PUBLISHED CVOTS OF HYPOGLYCEMIC AGENTS}

\section{Major adverse cardiovascular events}

Several CVOTs have recently been completed, most of which included similar primary outcome components: cardiovascular death, non-fatal MI, and non-fatal stroke. We summarize the published studies in Tables 1, 2 .

SGLT-2Is have been studied in three large placebo-controlled CVOTs in patients with T2DM. In the 2015 Empagliflozin Cardiovascular Outcome Event Trial in Type 2 Diabetes Mellitus Patients-Removing Excess Glucose (EMPA-REG) OUTCOME trial [29], there was a significant reduction in the primary outcome (MACE) for those receiving empagliflozin versus placebo (hazard ratio [HR], 0.86; 95\% confidence interval [CI], 0.74 to $0.99 ; P=0.04)$. A subsequent study of canagliflozin also showed reduced cardiovascular events in comparison to placebo (HR, 0.86 ; $95 \%$ CI, 0.75 to $0.97 ; P=0.02$ ) in the Canagliflozin Cardiovascular Assessment Study (CANVAS) program [13]. However, the largest trial on dapagliflozin, which enrolled 17,160 
Table 1. Overview of Recently Published Data on New Antidiabetic Drugs

\begin{tabular}{|c|c|c|c|c|c|c|}
\hline Drug class & Study name & Year & Age, yr & Underlying disease & Number & Follow-up, yr \\
\hline \multirow[t]{3}{*}{ SGLT2 inhibitors } & EMPA-REG (empagliflozin) & 2015 & $\geq 18$ & Established CVD & 7,020 & 3.1 \\
\hline & CANVAS-program (canagliflozin) & 2017 & $\geq 40$ & Pre-existing CVD or high CV risk & 10,142 & 1.5 \\
\hline & DECLARE-TIMI 53 (dapagliflozin) & 2018 & $\geq 40$ & High CV risk & 17,160 & 4.2 \\
\hline \multirow[t]{5}{*}{ GLP-1RA } & ELIXA (lixisenatide) & 2015 & $\geq 30$ & Patients with ACS before 180 days & 6,068 & 2.1 \\
\hline & LEADER (liraglutide) & 2016 & $\geq 50$ & $\begin{array}{l}\text { Established CVD or } \geq 60 \text { years with } \\
\geq 1 \mathrm{CV} \text { risk factor }\end{array}$ & 9,340 & 3.8 \\
\hline & SUSTAIN-6 (semaglutide) & 2016 & $\geq 50$ & $\begin{array}{l}\text { Established CVD or } \geq 60 \text { years with } \\
\geq 1 \mathrm{CV} \text { risk factor }\end{array}$ & 3,299 & 1.9 \\
\hline & EXSCEL (exenatide) & 2017 & $\geq 18$ & Pre-CVD $(73 \%)$ & 14,752 & 3.2 \\
\hline & HARMONY (albiglutide) & 2018 & $\geq 40$ & Pre-CVD & 9,643 & 1.6 \\
\hline
\end{tabular}

SGLT2, sodium-glucose cotransporter 2; EMPA-REG, Empagliflozin Cardiovascular Outcome Event Trial in Type 2 Diabetes Mellitus Patients-Removing Excess Glucose; CVD, cardiovascular disease; CANVAS, Canagliflozin Cardiovascular Assessment Study; CV, cardiovascular; DECLARETIMI 53, Dapagliflozin Effect on Cardiovascular Events-Thrombolysis in Myocardial Infarction 53; GLP-1RA, glucagon-like peptide 1 receptor agonist; ELIXA, Evaluation of Lixisenatide in Acute Coronary Syndrome; ACS, acute coronary syndrome; LEADER, Liraglutide Effect and Action in Diabetes: Evaluation of Cardiovascular Outcome Results; SUSTAIN-6, Semaglutide in Subjects with Type 2 Diabetes; EXSCEL, Exenatide Study of Cardiovascular Event Lowering; HARMONY, Albiglutide and Cardiovascular Outcomes in Patients with Type 2 Diabetes and Cardiovascular Disease.

Table 2. Primary Endpoint (Major Adverse Cardiovascular Events) of Completed Trials

\begin{tabular}{|c|c|c|c|c|c|}
\hline Drug class & Study name & Drug & HR & $95 \% \mathrm{CI}$ & Result \\
\hline \multirow[t]{3}{*}{ SGLT2 inhibitor } & EMPA-REG & Empagliflozin & 0.86 & $0.74-0.99$ & Positive \\
\hline & CANVAS-program & Canagliflozin & 0.86 & $0.75-0.97$ & Positive \\
\hline & DECLARE-TIMI 53 & Dapagliflozin & 0.93 & $0.84-1.03$ & Neutral \\
\hline \multirow[t]{5}{*}{ GLP-1RA } & ELIXA & Lixisenatide & 1.02 & $0.89-1.17$ & Neutral \\
\hline & LEADER & Liraglutide & 0.87 & $0.78-0.97$ & Positive \\
\hline & SUSTAIN-6 & Semaglutide & 0.74 & $0.58-0.95$ & Positive \\
\hline & EXSCEL & Exenatide & 0.91 & $0.83-1.00$ & Neutral \\
\hline & HARMONY & Albiglutide & 0.78 & $0.68-0.90$ & Positive \\
\hline
\end{tabular}

HR, hazard ratio; CI, confidence interval; SGLT2, sodium-glucose cotransporter 2; EMPA-REG, Empagliflozin Cardiovascular Outcome Event Trial in Type 2 Diabetes Mellitus Patients-Removing Excess Glucose; CANVAS, Canagliflozin Cardiovascular Assessment Study; DECLARE-TIMI 53, Dapagliflozin Effect on Cardiovascular Events-Thrombolysis in Myocardial Infarction 53; GLP-1RA, glucagon-like peptide 1 receptor agonist; ELIXA, Evaluation of Lixisenatide in Acute Coronary Syndrome; LEADER, Liraglutide Effect and Action in Diabetes: Evaluation of Cardiovascular Outcome Results; SUSTAIN-6, Semaglutide in Subjects with Type 2 Diabetes; EXSCEL, Exenatide Study of Cardiovascular Event Lowering; HARMONY, Albiglutide and Cardiovascular Outcomes in Patients with Type 2 Diabetes and Cardiovascular Disease.

patients, showed no significant changes in MACE rates [11]. However, direct comparison of MACE rates among CVOTs is challenging due to differences in the study populations. Although most of the patients in the EMPA-REG OUTCOME trial had established CVD, as did $72 \%$ of the population in the CANVAS program, the Dapagliflozin Effect on Cardiovascular Events-Thrombolysis in Myocardial Infarction (DECLARETIMI) 58 trial included more than 10,000 patients (59.4\%) and reported no obvious atherosclerotic cardiovascular events. A meta-analysis of CVOTs, including these three studies, showed that SGLT-2Is reduced MACE by $11 \%(\mathrm{HR}, 0.89 ; 95 \% \mathrm{CI}, 0.83$ to $0.96 ; P=0.0014$ ) [30].

There have been three studies on GLP-1RAs. In the Liraglutide Effect and Action in Diabetes: Evaluation of Cardiovascular Outcome Results (LEADER) trial, liraglutide reduced MACE by $13 \%$ (HR, $0.87 ; 95 \% \mathrm{CI}, 0.78$ to $0.97 ; P=0.01$ ), which was mainly driven by cardiovascular deaths. Semaglutide also significantly decreased MACE (HR, $0.74 ; 95 \%$ CI, 0.58 to 0.95; $P<0.001$ ); however, the decrease was driven by a reduction in non-fatal strokes, in contrast to the pattern observed for 
Table 3. CVOT Results According to Respective Outcomes

\begin{tabular}{|c|c|c|c|c|c|c|c|c|}
\hline \multirow{2}{*}{ Study name (drug) } & \multicolumn{2}{|c|}{ Cardiovascular death } & \multicolumn{2}{|c|}{ Nonfatal MI } & \multicolumn{2}{|c|}{ Nonfatal stroke } & \multicolumn{2}{|c|}{ Hospitalization for $\mathrm{HF}$} \\
\hline & $\operatorname{HR}(95 \% \mathrm{CI})$ & $P$ value & $\operatorname{HR}(95 \% \mathrm{CI})$ & $P$ value & $\operatorname{HR}(95 \% \mathrm{CI})$ & $P$ value & $\operatorname{HR}(95 \% \mathrm{CI})$ & $P$ value \\
\hline $\begin{array}{l}\text { EMPA-REG } \\
\text { (empagliflozin) }\end{array}$ & $0.62(0.49-0.77)$ & $<0.01$ & $0.87(0.70-1.09)$ & 0.23 & $1.24(0.92-1.67)$ & 0.16 & $0.65(0.50-0.84)$ & $<0.01$ \\
\hline $\begin{array}{l}\text { CANVAS-program } \\
\text { (canagliflozin) }\end{array}$ & $0.87(0.72-1.06)$ & & $0.85(0.69-1.05)$ & & $0.90(0.71-1.15)$ & & $0.67(0.52-0.87)$ & \\
\hline $\begin{array}{l}\text { DECLARE-TIMI } 53 \\
\text { (dapagliflozin) }\end{array}$ & $0.98(0.82-1.17)$ & & $0.89(0.77-1.01)$ & & $1.01(0.84-1.21)$ & & $0.73(0.61-0.88)$ & \\
\hline ELIXA (lixisenatide) & $0.98(0.78-1.22)$ & 0.85 & $1.03(0.87-1.22)$ & 0.71 & $1.12(0.79-1.58)$ & 0.54 & $0.96(0.75-1.23)$ & 0.75 \\
\hline LEADER (liraglutide) & $0.78(0.66-0.93)$ & $<0.01$ & $0.88(0.75-1.03)$ & 0.11 & $0.89(0.72-1.11)$ & 0.30 & $0.87(0.73-1.05)$ & 0.14 \\
\hline SUSTAIN-6 (semaglutide) & $0.98(0.65-1.48)$ & 0.92 & $0.74(0.51-1.08)$ & 0.12 & $0.61(0.38-0.99)$ & 0.04 & $1.11(0.77-1.61)$ & 0.57 \\
\hline EXSCEL (exenatide) & $0.88(0.76-1.02)$ & & $0.97(0.85-1.10)$ & & $0.85(0.70-1.03)$ & & $0.94(0.78-1.13)$ & \\
\hline HARMONY (albiglutide) & $0.93(0.73-1.19)$ & & $0.75(0.61-0.90)$ & & $0.86(0.66-1.14)$ & & - & \\
\hline
\end{tabular}

CVOT, cardiovascular outcome trial; HR, hazard ratio; CI, confidence interval; MI, myocardial infarction; HF, heart failure; EMPA-REG, Empagliflozin Cardiovascular Outcome Event Trial in Type 2 Diabetes Mellitus Patients-Removing Excess Glucose; CANVAS, Canagliflozin Cardiovascular Assessment Study; DECLARE-TIMI 53, Dapagliflozin Effect on Cardiovascular Events-Thrombolysis in Myocardial Infarction 53; ELIXA, Evaluation of Lixisenatide in Acute Coronary Syndrome; LEADER, Liraglutide Effect and Action in Diabetes: Evaluation of Cardiovascular Outcome Results; SUSTAIN-6, Semaglutide in Subjects with Type 2 Diabetes; EXSCEL, Exenatide Study of Cardiovascular Event Lowering; HARMONY, Albiglutide and Cardiovascular Outcomes in Patients with Type 2 Diabetes and Cardiovascular Disease.

liraglutide. In the Albiglutide and Cardiovascular Outcomes in Patients with Type 2 Diabetes and Cardiovascular Disease (Harmony Outcomes) trial, albiglutide reduced MACE by $22 \%$ (HR, $0.78 ; 95 \%$ CI, 0.68 to $0.90 ; P<0.001$ ), mainly driven by non-fatal MI.

\section{Atherosclerotic and hemodynamic CVD events in CVOTs Atherosclerotic events (cardiac death, MI, stroke)}

The LEADER trial showed that liraglutide, a GLP-1RA, is safe and effective for reducing the risk of cardiovascular events and death from cardiovascular-related causes [14]. Liraglutide decreased CVD mortality by $22 \%$, and death from any cause by $15 \%$. The rates of non-fatal MIs and non-fatal strokes were lower in the liraglutide group. In addition, a subgroup analysis revealed that older patients, and those with severe kidney disease or established CVD, may benefit more from liraglutide use [31]. Another GLP-1RA, semaglutide, decreased the primary outcome, which was driven by a reduction in non-fatal MI and stroke [32]. In the Harmony Outcomes, albiglutide significantly reduced non-fatal MI incidence by $25 \%$. A meta-analysis of GLP-1RAs reported a significant reduction of all-cause mortality and cardiovascular death [33]. Another recently published metaanalysis showed that the cardiovascular benefits of long-acting GLP-1RAs were more pronounced in Asian populations [34].

A few months before the LEADER trial results were released, the EMPA-REG OUTCOME trial reported that empagliflozin, an SGLT-2I, lowered the MACE rate in patients with T2DM and a high risk of CVD, mainly through a reduction in cardiovascular deaths (HR, 0.62; 95\% CI, 0.49 to 0.77) [29]. However, it did not reduce cardiovascular events, including MI and stroke. Other studies on SGLT-2Is also showed no reduction in atherosclerotic events compared to conventional therapy (Table 3). Although direct comparisons cannot be made between trials, the benefits of liraglutide observed in the LEADER trial differed from those of empagliflozin in the EMPA-REG OUTCOME trial. The cardiovascular benefits were seen within a few months after the initiation of empagliflozin treatment, but only after 12 to 16 months of liraglutide treatment.

\section{Heart failure}

SGLT-2Is, which lower hyperglycemia by increasing glycosuria, conferred benefits by reducing the rate of HHF ([empagliflozin: HR, 0.66; 95\% CI, 0.50 to 0.84]; [canagliflozin: HR, 0.87 ; $95 \% \mathrm{CI}, 0.52$ to 0.87 ]; [dapagliflozin: $\mathrm{HR}, 0.73$; $95 \% \mathrm{CI}$, 0.61 to 0.88$])[11,13,29]$. These positive effects of SGLT-2Is on HF have been confirmed by data from Comparative Effectiveness of Cardiovascular Outcomes in New Users of SGLT-2 Inhibitors (CVD-REAL) study (HR, $0.61 ; 95 \% \mathrm{CI}, 0.51$ to 0.73 ) and CVD-REAL2 study (HR, 0.64; 95\% CI, 0.50 to 0.82 ) $[35,36]$. In a meta-analysis including three large randomized tri- 
als, SGLT-2Is reduced the risk of cardiovascular death or HHF by $23 \%$ (HR, $0.77 ; 95 \% \mathrm{CI}, 0.71$ to $0.84 ; P<0.001$ ), regardless of prior CVD or HF.

SGLT-2Is did not reduce the risk of non-fatal MI or stroke in these studies, which suggests that their beneficial effects on CVD are related to hemodynamic effects. The specific mechanisms underlying the effects of empagliflozin on cardiovascular death are unknown. However, potential mechanisms include natriuretic effects [37], reductions of insulin levels and the insulin response to food intake, modulation of the renin-angiotensinaldosterone system [38], reductions in body weight and blood pressure $[39,40]$, and amelioration of the increases in sympathetic nervous activity thought to be responsible for HF [41-43].

The long-term effects of GLP-1RAs on HF have not been fully investigated. The Evaluation of Lixisenatide in Acute Coronary Syndrome (ELIXA) study, which was the first to investigate the effects of GLP-1RAs on CVD safety, showed no effect on HHF even in subgroups of patients who previously had HF [44]. The LEADER study also showed that liraglutide had no effect on HHF (HR, 0.87 ; 95\% CI, 0.73 to 1.05 ), despite a significant reduction in the composite primary outcome of cardiovascular death, non-fatal MI, and non-fatal stroke (HR, 0.87; $95 \% \mathrm{CI}, 0.78$ to 0.97 ) [14].

\section{Renal outcomes}

Hypertrophy of the proximal tubule (PT) commonly occurs in the early stages of diabetes [45]. PT growth is accompanied by an increase in glucose reabsorption capacity and sodium reabsorption, and can be associated with higher renal expression of SGLT-2 [46,47]. The increase in active tubular transport enhances oxygen consumption, and hypoxia of the renal cortex is an important factor in the development of renal interstitial fibrosis and progression to CKD $[48,49]$. The EMPA-REG OUTCOME trial also investigated renal outcomes in patients with T2DM who were at high risk of cardiovascular events, with an estimated glomerular filtration rate (eGFR) of $\geq 30 \mathrm{~mL} / \mathrm{min} / 1.73 \mathrm{~m}^{2}$. Compared with placebo, empagliflozin was associated with a significant risk reduction for a composite renal microvascular outcome (HR, $0.61 ; 95 \% \mathrm{CI}, 0.53$ to $0.70 ; P<0.001$ ), defined as progression to macroalbuminuria; a doubling of the serum creatinine level (with an eGFR $\leq 45 \mathrm{~mL} / \mathrm{min} / 1.73 \mathrm{~m}^{2}$ ); the initiation of renal replacement therapy, or death from renal disease [50]. This protective effect was sustained in patients with $\mathrm{CKD}$, defined as an eGFR $<60 \mathrm{~mL} / \mathrm{min} / 1.73 \mathrm{~m}^{2}$ and macroalbuminuria at baseline. Canagliflozin reduced the likelihood of progression to albuminuria and increased the likelihood of regres- sion of albuminuria (HR, $0.73 ; 95 \% \mathrm{CI}, 0.67$ to 0.79 ), and also showed a renal protective effect in the composite renal outcome, which comprised a $40 \%$ reduction in eGFR sustained for at least two consecutive measures, renal replacement therapy, or death from renal disease (HR, $0.60 ; 95 \% \mathrm{CI}, 0.47$ to 0.77 ). In the DECLARE-TIMI 58 trial, a renal protective effect of dapagliflozin was also observed (HR, 0.76 ; $95 \%$ CI, 0.67 to 0.87 ).

Early in empagliflozin treatment (week 4), the eGFR slightly decreased, whereas the placebo group showed no significant change; however, during the long-term follow-up, the eGFR remained stable and empagliflozin treatment was associated with a lower annual decrease in the eGFR $\left(0.19 \pm 0.11 \mathrm{~mL} / \mathrm{min} / 1.73 \mathrm{~m}^{2}\right.$ vs. $1.67 \pm 0.13 \mathrm{~mL} / \mathrm{min} / 1.73 \mathrm{~m}^{2}$ ). Canagliflozin also reduced the eGFR during week 3 to 6 , but it stabilized over the remainder of the study period. Dapagliflozin induced a greater reduction in the eGFR after 1 week than placebo, and the eGFR subsequently increased to near-baseline values after 24 weeks and remained stable until the end of treatment at 102 weeks. At that time, the glomerular filtration rate (GFR) was not significantly different between the treatment and placebo control groups [51]. The acute GFR-lowering effect of SGLT-2Is is associated with increased delivery of glucose and sodium to the downstream macula densa [52,53]. The increased hydrostatic and osmotic pressure caused by non-resorbed glucose can decrease glomerular filtration through the tubuloglomerular feedback (TGF) system. The role of the TGF system is to stabilize salt and fluid delivery to the further distal nephron. The reduced fluid delivery to the distal tubule lowers the tubular back pressure in the Bowman space, which increases the effective glomerular filtration pressure $[53,54]$. On the contrary, increased sodium, chloride, and glucose delivery to the downstream macula densa by SGLT-2 inhibition enhances the tone of the afferent arteriole and lowers GFR by reducing glomerular plasma flow and intraglomerular pressure. In addition, the osmotic effect of non-reabsorbed glucose increases the tubular back pressure, thereby reducing the effective filtration pressure [55]. It may be more than a coincidence that the time course of the eGFR decline after SGLT-2 blockade is similar to that observed after angiotensin blockade, which also causes an immediate functional decline in the GFR due to reduced intraglomerular pressure, but slows disease progression. In other words, the initial and functional decrease in the eGFR in response to blockade of angiotensin or SGLT-2 may help to preserve kidney function during long-term follow-up.

\section{The CVD-REAL and CVD-REAL2 clinical trials}

Two studies confirmed the cardioprotective effect of SGLT-2Is 
in real-world practice $[35,36]$. In the CVD-REAL study, treatment with SGLT-2Is reduced the relative risk of HHF by $39 \%$ and the risk of all-cause death by $51 \%$. Although most previous CVOTs included patients with established cardiovascular, $87 \%$ of patients did not have CVD in the CVD-REAL study. Thus, the CVD-REAL study was the first study to show a class effect of SGLT-2Is against CVD in patients with a lower risk of the disease. However, the CVD-REAL study was limited to the United States and Europe. A study of patients from Asia Pacific, the Middle East, and North America, published in 2018, included about 330,000 Koreans [35]. The results, based on what can be regarded as "real-world data," showed that SGLT-2Is reduced the all-cause death risk by $49 \%$ and the HHF rate by $36 \%$. Death due to HHF was reduced by $40 \%$. The risks of MI and stroke were also reduced by $19 \%$ and $32 \%$, respectively. When restricted to Koreans, there was a $28 \%$ risk reduction of all-cause death and a $13 \%$ reduction of HHF.

Real-world data extracted from the Korean Health Insurance Review and Assessment Service database were published recently [12]. SGLT-2Is reduced HHF by $34 \%$ (95\% CI, 0.58 to $0.75)$ compared with dipeptidyl peptidase- 4 inhibitors. The effect was present at 30 days after initiating SGLT-2I treatment among patients with established CVD, and persisted at a 3-year follow-up in patients without CVD. According to these realworld data, the cardiovascular benefits of SGLT-2Is appear to exist regardless of race and CVD risk.

However, since these data were drawn from a retrospective study, we can only mention their relevance based on the conclusions of the research, as there is a limit to the degree to which these findings can be directly extrapolated as indicative of racial differences or class effects. In addition, it was pointed out that the results may have been somewhat exaggerated due to immortal time bias. Therefore, a well-designed randomized controlled trial is needed to investigate these issues.

\section{WHICH AGENTS ARE BENEFICIAL IN PATIENTS WITH DIABETES AT AN INCREASED RISK OF CVD?}

\section{GLP-1RAs for treating atherosclerosis}

GLP-1RAs are currently delivered via subcutaneous injection. These agents stimulate insulin secretion and reduce glucagon secretion in a glucose-dependent manner, thus enhancing satiety and promoting weight loss [56,57]. Liraglutide and semaglutide have been shown to improve cardiovascular outcomes, as discussed above. A recently published meta-analysis including the
LEADER (liraglutide), Semaglutide in Subjects with Type 2 Diabetes (SUSTAIN-6; semaglutide), and Exenatide Study of Cardiovascular Event Lowering (EXSCEL; exenatide) trials showed that GLP-1RAs reduced the risks of MACE and cardiovascular death in patients with T2DM. A recently published meta-analysis showed particularly favorable trends in Asian populations regarding the beneficial effects of GLP-1RAs on cardiovascular outcomes [34]. Although the mechanism of the differential cardiovascular effect according to race is unclear, the authors suggested the possibility that endogenous GLP-1 levels are lower in Asian populations [58,59], so exogenous GLP1RAs could compensate for this deficiency. Second, better glycemic control by GLP-IRAs, especially in Asian populations, could reduce the incidence of cardiovascular events [60].

The positive effect of GLP-1RAs on atherosclerotic disease could be partly explained by the results of preclinical studies showing that GLP-1RAs are present in cardiac myocytes, the vascular endothelium, and arterial smooth muscle cells [61], as well as in the pancreas and gut, and that GLP-1RAs show cardioprotective effects through various mechanisms, including reduction of atherosclerotic lesions [62], improvement of endothelial function [63] and ventricular systolic function [64], reduction of infarct size, and improvement of left ventricular function after coronary ischemia [65].

\section{Effect of SGLT-2Is on heart failure and CKD}

In the EMPA-REG OUTCOME trial, there was a significant reduction in the primary outcome (three-point MACE), the risk of cardiovascular death for those receiving empagliflozin, and the risk of HHF. The effects on both cardiovascular death and HHF were apparent immediately after the drug intervention. Similar cardioprotective effects, especially a reduction of HHF by SGLT-2Is, were seen in a meta-analysis including three major randomized controlled trials [30].

The class effects of SGLT-2Is in reducing the risk of cardiovascular death and HHF may be explained principally by their apparent ability to alter cardiac physiology, and specifically to reduce the cardiac workload and myocardial oxygen demand $\left(\mathrm{MVO}_{2}\right)$ by lowering blood pressure and improving aortic compliance and ventricular arterial coupling [66].

The ability of SGLT-2Is to lower blood pressure is also important for reducing the cardiac workload. Modest reductions in systolic (3 to $5 \mathrm{~mm} \mathrm{Hg}$ ) and diastolic (2 to $3 \mathrm{~mm} \mathrm{Hg}$ ) blood pressure without an increase in heart rate have been reported using canagliflozin, dapagliflozin, and empagliflozin [40,67]. Previous analyses have reported beneficial effects of even small reduc- 
tions in blood pressure ( $\leq 3 \mathrm{~mm} \mathrm{Hg}$ ) in decreasing cardiovascular events, such as coronary heart disease and HF [68,69], via unloading the ventricle, reducing ventricular wall tension, and making the ventricle smaller, which in turn reduces $\mathrm{MVO}_{2}$ and decreases the power needed to increase the stroke volume. Unloading the ventricle also lowers the pressure on the intracardial surface and helps to decrease microcellular ischemia, such that ventricular arterial coupling is improved and the heart functions with greater efficiency, using less energy each time it pumps.

In addition, in diabetic patients with $\mathrm{HF}$ or CKD, regardless of previous CVD, the use of SGLT-2Is reduced CKD progression and CVD events [29,70].

\section{Risk stratification and the updated 2018 ADA/EASD guidelines}

Although the development of CVD is closely associated with T2DM, not all T2DM patients have the same level of risk. Therefore, risk stratification is needed when choosing a hypoglycemic agent to prevent or decrease the risk of CVD. Generally, cardiovascular risk increases with diabetes duration, and it is also affected by other comorbidities such as hypertension, dyslipidemia, obesity, and CKD [71].

The results of both the LEADER and EMPA-REG OUTCOME trials represent a clinical breakthrough in the pharmacologic management of patients with T2DM, suggesting a paradigm shift toward use of either empagliflozin or liraglutide in addition to metformin, or even as a first-line therapy if there is preexisting atherosclerotic CVD or a high risk thereof. According to the updated guidelines, management of hyperglycemia should focus on a patient-centered strategy. Metformin remains the foundation therapy, and if hemoglobin A1c levels are $\geq 1.5 \%$ above the target for a given individual, early combined use of metformin with an additional agent should be considered. If a patient has established atherosclerotic CVD, HF, or CKD, then SGLT2Is or GLP-1RAs, which have proven cardiovascular benefits, are recommended. For patients without atherosclerotic CVD, HF, or CKD, treatment should focus on the patient's needs and characteristics, including weight, risk of hypoglycemia, and economic circumstances. In addition, GLP-1R agonists are preferred as the first-line injectable therapy over insulin in most cases.

\section{CONCLUSIONS}

In recently published representative trials, both GLP-1RAs and SGLT-2Is showed favorable cardioprotective effects and safety in high-risk patients with T2DM. This points to a paradigm shift toward a GLP-1RA- or SGLT-2I-centered strategy in diabetic patients with established CVD.

GLP-1RAs have robust benefits for MACE and cardiac death, and showed a favorable effect on cardiovascular outcomes in Asians. In addition, SGLT-2Is significantly reduced HHF and progression of renal disease, regardless of underlying CVD. Therefore, studies on the different mechanisms and effects of the two drugs, according to underlying disease, race, and demographic characteristics, are needed. In addition, the effects of these drugs on CVD outcomes in patients at a lower risk of CVD, and as primary prevention, remain known. The LEADER trial did not show a reduction in the primary composite outcome in patients without established CVD (19\% of the cohort), and the EMPA-REG OUTCOME trial only included patients with pre-existing CVD (99\% of the cohort). Therefore, it is necessary to determine whether the CVD-lowering effects of these two drugs can be reproduced in patients without CVD.

\section{CONFLICTS OF INTEREST}

No potential conflict of interest relevant to this article was reported.

\section{ACKNOWLEDGMENTS}

This work was supported by Priority Research Centers Program through the National Research Foundation of Korea (NRF) funded by the Ministry of Education (2010-0020224).

\section{ORCID}

Jong Chul Won https://orcid.org/0000-0002-2219-4083

\section{REFERENCES}

1. Guariguata L, Whiting DR, Hambleton I, Beagley J, Linnenkamp U, Shaw JE. Global estimates of diabetes prevalence for 2013 and projections for 2035. Diabetes Res Clin Pract 2014;103:137-49.

2. Rawshani A, Rawshani A, Franzen S, Eliasson B, Svensson AM, Miftaraj M, et al. Mortality and cardiovascular disease in type 1 and type 2 diabetes. N Engl J Med 2017;376:140718.

3. Preis SR, Hwang SJ, Coady S, Pencina MJ, D'Agostino RB Sr, Savage PJ, et al. Trends in all-cause and cardiovascular disease mortality among women and men with and without 
diabetes mellitus in the Framingham Heart Study, 1950 to 2005. Circulation 2009;119:1728-35.

4. Wong HK, Ong KL, Cheung CL, Cheung BM. Utilization of glucose, blood pressure, and lipid lowering medications among people with type II diabetes in the United States, 1999-2010. Ann Epidemiol 2014;24:516-21.

5. Holman RR, Paul SK, Bethel MA, Matthews DR, Neil HA. 10-Year follow-up of intensive glucose control in type 2 diabetes. N Engl J Med 2008;359:1577-89.

6. ACCORD Study Group, Cushman WC, Evans GW, Byington RP, Goff DC Jr, Grimm RH Jr, et al. Effects of intensive blood-pressure control in type 2 diabetes mellitus. N Engl $\mathbf{J}$ Med 2010;362:1575-85.

7. ADVANCE Collaborative Group, Patel A, MacMahon S, Chalmers J, Neal B, Billot L, et al. Intensive blood glucose control and vascular outcomes in patients with type 2 diabetes. N Engl J Med 2008;358:2560-72.

8. Nissen SE, Wolski K. Effect of rosiglitazone on the risk of myocardial infarction and death from cardiovascular causes. N Engl J Med 2007;356:2457-71.

9. Food and Drug Administration (Center for Drug Evaluation and Research). Guidance for industry: diabetes mellitus: evaluating cardiovascular risk in new antidiabetic therapies to treat type 2 diabetes [Internet]. Silver Spring: FDA; 2008 [cited 2019 Mar 25]. Available from: http://www.fda.gov/ downloads/drugs/guidancecomplianceregulatoryinformation/guidances/ucm071627.pdf.

10. Zheng SL, Roddick AJ, Aghar-Jaffar R, Shun-Shin MJ, Francis D, Oliver N, et al. Association between use of sodium-glucose cotransporter 2 inhibitors, glucagon-like peptide 1 agonists, and dipeptidyl peptidase 4 inhibitors with allcause mortality in patients with type 2 diabetes: a systematic review and meta-analysis. JAMA 2018;319:1580-91.

11. Wiviott SD, Raz I, Bonaca MP, Mosenzon O, Kato ET, Cahn A, et al. Dapagliflozin and cardiovascular outcomes in type 2 diabetes. N Engl J Med 2019;380:347-57.

12. Kim YG, Han SJ, Kim DJ, Lee KW, Kim HJ. Association between sodium glucose co-transporter 2 inhibitors and a reduced risk of heart failure in patients with type 2 diabetes mellitus: a real-world nationwide population-based cohort study. Cardiovasc Diabetol 2018;17:91.

13. Neal B, Perkovic V, Mahaffey KW, de Zeeuw D, Fulcher G, Erondu N, et al. Canagliflozin and cardiovascular and renal events in type 2 diabetes. N Engl J Med 2017;377:644-57.

14. Marso SP, Daniels GH, Brown-Frandsen K, Kristensen P, Mann JF, Nauck MA, et al. Liraglutide and cardiovascular outcomes in type 2 diabetes. N Engl J Med 2016;375:311-22.

15. Davies MJ, D'Alessio DA, Fradkin J, Kernan WN, Mathieu C, Mingrone G, et al. Management of hyperglycemia in type 2 diabetes, 2018. A consensus report by the American Diabetes Association (ADA) and the European Association for the Study of Diabetes (EASD). Diabetes Care 2018;41:2669701.

16. Koo BK, Lee CH, Yang BR, Hwang SS, Choi NK. The incidence and prevalence of diabetes mellitus and related atherosclerotic complications in Korea: a National Health Insurance database study. PLoS One 2014;9:e110650.

17. Kang YM, Cho YK, Lee SE, Park JY, Lee WJ, Kim YJ, et al. Cardiovascular diseases and life expectancy in adults with type 2 diabetes: a Korean national sample cohort study. J Clin Endocrinol Metab 2017;102:3443-51.

18. Ross R. Atherosclerosis: an inflammatory disease. N Engl J Med 1999;340:115-26.

19. Khunti K, Kosiborod M, Ray KK. Legacy benefits of blood glucose, blood pressure and lipid control in individuals with diabetes and cardiovascular disease: time to overcome multifactorial therapeutic inertia? Diabetes Obes Metab 2018; 20:1337-41.

20. Gaede P, Oellgaard J, Carstensen B, Rossing P, Lund-Andersen $\mathrm{H}$, Parving HH, et al. Years of life gained by multifactorial intervention in patients with type 2 diabetes mellitus and microalbuminuria: 21 years follow-up on the Steno-2 randomised trial. Diabetologia 2016;59:2298-307.

21. Riddle MC, Gerstein HC, Holman RR, Inzucchi SE, Zinman $\mathrm{B}$, Zoungas S, et al. A1C targets should be personalized to maximize benefits while limiting risks. Diabetes Care 2018; 41:1121-4.

22. Task Force on diabetes, pre-diabetes, and cardiovascular diseases of the European Society of Cardiology (ESC); European Association for the Study of Diabetes (EASD), Ryden L, Grant PJ, Anker SD, Berne C, et al. ESC guidelines on diabetes, pre-diabetes, and cardiovascular diseases developed in collaboration with the EASD: summary. Diab Vasc Dis Res 2014;11:133-73.

23. Lee JH, Lim NK, Cho MC, Park HY. Epidemiology of heart failure in Korea: present and future. Korean Circ J 2016;46: 658-64.

24. Rosano GM, Fini M, Caminiti G, Barbaro G. Cardiac metabolism in myocardial ischemia. Curr Pharm Des 2008;14: 2551-62.

25. Ko TH, Marquez JC, Kim HK, Jeong SH, Lee S, Youm JB, et al. Resistance exercise improves cardiac function and mi- 
tochondrial efficiency in diabetic rat hearts. Pflugers Arch 2018;470:263-75.

26. Home PD, Pocock SJ, Beck-Nielsen H, Curtis PS, Gomis R, Hanefeld M, et al. Rosiglitazone evaluated for cardiovascular outcomes in oral agent combination therapy for type 2 diabetes (RECORD): a multicentre, randomised, open-label trial. Lancet 2009;373:2125-35.

27. Elder DH, Singh JS, Levin D, Donnelly LA, Choy AM, George J, et al. Mean HbA1c and mortality in diabetic individuals with heart failure: a population cohort study. Eur J Heart Fail 2016;18:94-102.

28. Boussageon R, Bejan-Angoulvant T, Saadatian-Elahi M, Lafont S, Bergeonneau C, Kassai B, et al. Effect of intensive glucose lowering treatment on all cause mortality, cardiovascular death, and microvascular events in type 2 diabetes: meta-analysis of randomised controlled trials. BMJ 2011; 343:d4169.

29. Zinman B, Wanner C, Lachin JM, Fitchett D, Bluhmki E, Hantel S, et al. Empagliflozin, cardiovascular outcomes, and mortality in type 2 diabetes. N Engl J Med 2015;373:2117-28.

30. Zelniker TA, Wiviott SD, Raz I, Im K, Goodrich EL, Bonaca MP, et al. SGLT2 inhibitors for primary and secondary prevention of cardiovascular and renal outcomes in type 2 diabetes: a systematic review and meta-analysis of cardiovascular outcome trials. Lancet 2019;393:31-9.

31. Roder ME. Major adverse cardiovascular event reduction with GLP-1 and SGLT2 agents: evidence and clinical potential. Ther Adv Chronic Dis 2018;9:33-50.

32. Marso SP, Bain SC, Consoli A, Eliaschewitz FG, Jodar E, Leiter LA, et al. Semaglutide and cardiovascular outcomes in patients with type 2 diabetes. N Engl J Med 2016;375: 1834-44.

33. Gargiulo P, Savarese G, D’Amore C, De Martino F, Lund LH, Marsico F, et al. Efficacy and safety of glucagon-like peptide-1 agonists on macrovascular and microvascular events in type 2 diabetes mellitus: a meta-analysis. Nutr Metab Cardiovasc Dis 2017;27:1081-8.

34. Kang YM, Cho YK, Lee J, Lee SE, Lee WJ, Park JY, et al. Asian subpopulations may exhibit greater cardiovascular benefit from long-acting glucagon-like peptide 1 receptor agonists: a meta-analysis of cardiovascular outcome trials. Diabetes Metab J 2018 Dec 27 [Epub]. https://doi.org/10.4093/ dmj.2018.0070.

35. Kosiborod M, Lam CSP, Kohsaka S, Kim DJ, Karasik A, Shaw J, et al. Cardiovascular events associated with SGLT-2 inhibitors versus other glucose-lowering drugs: the CVD-
REAL study. J Am Coll Cardiol 2018;71:2628-39.

36. Kosiborod M, Cavender MA, Fu AZ, Wilding JP, Khunti K, Holl RW, et al. Lower risk of heart failure and death in patients initiated on sodium-glucose cotransporter-2 inhibitors versus other glucose-lowering drugs: the CVD-REAL study (comparative effectiveness of cardiovascular outcomes in new users of sodium-glucose cotransporter-2 inhibitors). Circulation 2017;136:249-59.

37. Tanaka H, Takano K, Iijima H, Kubo H, Maruyama N, Hashimoto T, et al. Factors affecting canagliflozin-induced transient urine volume increase in patients with type 2 diabetes mellitus. Adv Ther 2017;34:436-51.

38. Ansary TM, Nakano D, Nishiyama A. Diuretic effects of sodium glucose cotransporter 2 inhibitors and their influence on the renin-angiotensin system. Int J Mol Sci 2019;20:E629.

39. Tikkanen I, Narko K, Zeller C, Green A, Salsali A, Broedl UC, et al. Empagliflozin reduces blood pressure in patients with type 2 diabetes and hypertension. Diabetes Care 2015; 38:420-8.

40. Tikkanen I, Chilton R, Johansen OE. Potential role of sodium glucose cotransporter 2 inhibitors in the treatment of hypertension. Curr Opin Nephrol Hypertens 2016;25:81-6.

41. Pham SV, Chilton RJ. EMPA-REG OUTCOME: the cardiologist's point of view. Am J Cardiol 2017;120:S53-8.

42. Rahman A, Fujisawa Y, Nakano D, Hitomi H, Nishiyama A. Effect of a selective SGLT2 inhibitor, luseogliflozin, on circadian rhythm of sympathetic nervous function and locomotor activities in metabolic syndrome rats. Clin Exp Pharmacol Physiol 2017;44:522-5.

43. Wan N, Rahman A, Hitomi H, Nishiyama A. The effects of sodium-glucose cotransporter 2 inhibitors on sympathetic nervous activity. Front Endocrinol 2018;9:421.

44. Pfeffer MA, Claggett B, Diaz R, Dickstein K, Gerstein HC, Kober LV, et al. Lixisenatide in patients with type 2 diabetes and acute coronary syndrome. N Engl J Med 2015;373:224757.

45. Vallon V. The proximal tubule in the pathophysiology of the diabetic kidney. Am J Physiol Regul Integr Comp Physiol 2011;300:R1009-22.

46. Wang XX, Levi J, Luo Y, Myakala K, Herman-Edelstein M, Qiu L, et al. SGLT2 protein expression is increased in human diabetic nephropathy: SGLT2 protein inhibition decreases renal lipid accumulation, inflammation, and the development of nephropathy in diabetic mice. J Biol Chem 2017;292:5335-48.

47. Vallon V, Rose M, Gerasimova M, Satriano J, Platt KA, Ko- 
epsell H, et al. Knockout of Na-glucose transporter SGLT2 attenuates hyperglycemia and glomerular hyperfiltration but not kidney growth or injury in diabetes mellitus. Am J Physiol Renal Physiol 2013;304:F156-67.

48. Hansell P, Welch WJ, Blantz RC, Palm F. Determinants of kidney oxygen consumption and their relationship to tissue oxygen tension in diabetes and hypertension. Clin Exp Pharmacol Physiol 2013;40:123-37.

49. Vallon V, Komers R. Pathophysiology of the diabetic kidney. Compr Physiol 2011;1:1175-232.

50. Wanner C, Inzucchi SE, Lachin JM, Fitchett D, von Eynatten M, Mattheus M, et al. Empagliflozin and progression of kidney disease in type 2 diabetes. N Engl J Med 2016;375:32334.

51. Kohan DE, Fioretto P, Johnsson K, Parikh S, Ptaszynska A, Ying L. The effect of dapagliflozin on renal function in patients with type 2 diabetes. J Nephrol 2016;29:391-400.

52. Thomson SC, Rieg T, Miracle C, Mansoury H, Whaley J, Vallon V, et al. Acute and chronic effects of SGLT2 blockade on glomerular and tubular function in the early diabetic rat. Am J Physiol Regul Integr Comp Physiol 2012;302:R75-83.

53. Vallon V, Richter K, Blantz RC, Thomson S, Osswald H. Glomerular hyperfiltration in experimental diabetes mellitus: potential role of tubular reabsorption. J Am Soc Nephrol 1999;10:2569-76.

54. Hallow KM, Gebremichael Y, Helmlinger G, Vallon V. Primary proximal tubule hyperreabsorption and impaired tubular transport counterregulation determine glomerular hyperfiltration in diabetes: a modeling analysis. Am J Physiol Renal Physiol 2017;312:F819-35.

55. Nespoux J, Vallon V. SGLT2 inhibition and kidney protection. Clin Sci 2018;132:1329-39.

56. Thrasher J. Pharmacologic management of type 2 diabetes mellitus: available therapies. Am J Med 2017;130:S4-17.

57. Karagiannis T, Liakos A, Bekiari E, Athanasiadou E, Paschos P, Vasilakou D, et al. Efficacy and safety of onceweekly glucagon-like peptide 1 receptor agonists for the management of type 2 diabetes: a systematic review and meta-analysis of randomized controlled trials. Diabetes Obes Metab 2015;17:1065-74.

58. Zhang F, Tang X, Cao H, Lu Q, Li N, Liu Y, et al. Impaired secretion of total glucagon-like peptide-1 in people with impaired fasting glucose combined impaired glucose tolerance. Int J Med Sci 2012;9:574-81.

59. Yabe D, Kuroe A, Lee S, Watanabe K, Hyo T, Hishizawa M, et al. Little enhancement of meal-induced glucagon-like peptide 1 secretion in Japanese: comparison of type 2 diabetes patients and healthy controls. J Diabetes Investig 2010;1: 56-9.

60. Kim YG, Hahn S, Oh TJ, Park KS, Cho YM. Differences in the HbAlc-lowering efficacy of glucagon-like peptide-1 analogues between Asians and non-Asians: a systematic review and meta-analysis. Diabetes Obes Metab 2014;16:900-9.

61. Meier JJ. GLP-1 receptor agonists for individualized treatment of type 2 diabetes mellitus. Nat Rev Endocrinol 2012;8: 728-42.

62. Nagashima M, Watanabe T, Terasaki M, Tomoyasu M, Nohtomi K, Kim-Kaneyama J, et al. Native incretins prevent the development of atherosclerotic lesions in apolipoprotein $\mathrm{E}$ knockout mice. Diabetologia 2011;54:2649-59.

63. Ceriello A, Esposito K, Testa R, Bonfigli AR, Marra M, Giugliano $\mathrm{D}$. The possible protective role of glucagon-like peptide 1 on endothelium during the meal and evidence for an "endothelial resistance" to glucagon-like peptide 1 in diabetes. Diabetes Care 2011;34:697-702.

64. Nikolaidis LA, Mankad S, Sokos GG, Miske G, Shah A, Elahi D, et al. Effects of glucagon-like peptide-1 in patients with acute myocardial infarction and left ventricular dysfunction after successful reperfusion. Circulation 2004;109: 962-5.

65. Noyan-Ashraf MH, Momen MA, Ban K, Sadi AM, Zhou YQ, Riazi AM, et al. GLP-1R agonist liraglutide activates cytoprotective pathways and improves outcomes after experimental myocardial infarction in mice. Diabetes 2009;58: 975-83.

66. Chilton RJ, Gullestad L, Fitchett D, Inzucchi SE, Mattheus $\mathrm{M}$, Woerle HJ, et al. Empagliflozin reduces markers of arterial stiffness, vascular resistance and cardiac workload in EMPA-REG OUTCOME. Circulation 2018;134:A13520.

67. Abdul-Ghani M, Del Prato S, Chilton R, DeFronzo RA. SGLT2 inhibitors and cardiovascular risk: lessons learned from the EMPA-REG OUTCOME study. Diabetes Care 2016;39:717-25.

68. Hardy ST, Loehr LR, Butler KR, Chakladar S, Chang PP, Folsom AR, et al. Reducing the blood pressure-related burden of cardiovascular disease: impact of achievable improvements in blood pressure prevention and control. J Am Heart Assoc 2015;4:e02276.

69. Cook NR, Cohen J, Hebert PR, Taylor JO, Hennekens CH. Implications of small reductions in diastolic blood pressure for primary prevention. Arch Intern Med 1995;155:701-9.

70. Boussageon R, Supper I, Bejan-Angoulvant T, Kellou N, 
Cucherat M, Boissel JP, et al. Reappraisal of metformin efficacy in the treatment of type 2 diabetes: a meta-analysis of randomised controlled trials. PLoS Med 2012;9:e1001204.
71. Saydah SH, Eberhardt MS, Loria CM, Brancati FL. Age and the burden of death attributable to diabetes in the United States. Am J Epidemiol 2002;156:714-9. 